\title{
Qualidade do Gasto Público em Educação Fundamental: uma análise de eficiência dos municípios paulistas
}

\author{
Public Spending Quality in Fundamental Education: an efficiency analysis in São \\ Paulo's municipalities
}

\author{
Alessandra Ayumi S. B. de S. Kakihara ${ }^{1}$, Vanderléia de Souza da Silva ${ }^{1 *}$, Johan Hendrik Poker Junior ${ }^{1}$ \\ ${ }^{1}$ Universidade Estadual de Campinas (UNICAMP) - São Paulo, Brasil.
}

\section{NFO ARTIGO \\ Palavras-chave: \\ Educação fundamental, Eficiência, \\ Qualidade do gasto público.}

\section{ARTICLE INFO}

\section{Keywords:}

Elementary

education,

Efficiency,

Quality public

spending.

\begin{abstract}
RESU MO
O estudo analisou a eficiência do gasto público em educação fundamental realizados nos municípios paulistas que compreendem as diretorias de ensino de Americana, Botucatu, Capivari, Limeira, Piracicaba, Pirassununga, São Carlos e Sumaré, no período entre 2009 a 2013, devido à disponibilidade de dados. Em uma segunda etapa, buscou-se a identificação de variáveis que interferem na eficiência resultada. Para isso, foi aplicada a metodologia de Análise Envoltória de Dados (DEA) e Regressão Linear Múltipla (RLM). Os resultados da DEA apontaram os municípios eficientes em cada ano estudado e que alguns municípios demonstram a necessidade imediata de mudanças na gestão educacional. A RLM apontou as variáveis que interferem nessa eficiência foram: quantidade de população rural, quantidade de alunos por turma, docentes com nível superior e a regularidade destes no processo educacional. Assim, consegue-se expor ao gestor público a necessidade de dirimir a interferência destes fatores, obtendo melhoria da aplicação dos recursos em educação e, consequentemente, melhoria na qualidade do ensino.
\end{abstract}

\begin{abstract}
A B S T R A C T
The study analyzed the efficiency of public spending on basic education carried out in São Paulo municipalities that comprise the boards of education of Americana, Botucatu, Capivari, Limeira, Piracicaba, Pirassununga, São Carlos and Sumaré, in the period between 2009 and 2013, due to data availability. In a second step, we sought to identify variables that interfere with the resulting efficiency. For that, the Data Envelopment Analysis (DEA) and Multiple Linear Regression (MLR) methodology was applied. The DEA results showed efficient municipalities in each year studied and that some municipalities demonstrate the immediate need for changes in educational management. The MLR pointed out the variables that interfere in this efficiency were: number of rural population, number of students per class, teachers with higher education and their regularity in the educational process. Thus, it is possible to expose to the public manager the need to resolve the interference of these factors, obtaining an improvement in the application of resources in education and, consequently, improving the quality of teaching.
\end{abstract}

\footnotetext{
* Correspondência para autor: alessandra_savassi@hotmail.com (Kakihara, A.A.S.B.S.), vanderleia_adm@hotmail.com (da Silva, V.S.) (0000-0003-0525-9137), johan.poker@fca.unicamp.br (Poker JR., J.H.).
}

DOI: doi.org/10.21714/1679-1827-2020.v18.n1.p136-153

1679-1827 (C) 2019 Gest@o.org. 


\section{Introdução}

Os recursos da educação no Brasil são oriundos dos tributos recolhidos pelos governos municipal, estadual e federal, sendo função dos estados e municípios a aplicação destes no Ensino Fundamental. Pensar que quanto maior for o repasse, melhor será a qualidade de ensino é uma simplificação perigosa do problema, em especial, pois o aumento das receitas é inviável, em face da alta carga tributária de todos os níveis de governo (ZOGHBI et al., 2011). Nesse sentido, a qualidade do gasto público importa, pois maiores gastos não significam necessariamente aumento de qualidade e podem, ao contrário, estar afetando de maneira negativa a boa gestão de recursos públicos. Há, portanto, uma relação custo-benefício que precisa ser considerada (POKER JR. et al., 2013).

O sistema de educacional brasileiro é dividido em Educação Básica e Educação Superior, onde o primeiro compreende a Educação Infantil (EI), Ensino Fundamental (EF) e Ensino Médio (EM); e o segundo, a Graduação e Pós-Graduação. O foco do ensino é o desenvolvimento da capacidade de aprendizado do aluno, por meio do domínio da leitura, escrita e do cálculo. Este tem a duração de 9 anos, dividido em Anos Iniciais (AI), que compreende as séries de $1^{\mathrm{a}}$. a $4^{\mathrm{a}}$. ( $1^{\mathrm{o}}$. ao $5^{\mathrm{o}}$. ano); e Anos Finais (AF), que compreende as séries de $5^{\mathrm{a}}$. a $8^{\mathrm{a}}$. ( $6^{\circ}$. ao $9^{\circ}$. ano), sendo este o foco de análise no presente estudo (PORTAL BRASIL, 2014).

Fazendo uma breve análise do valor destinado ao investimento público direto em educação por estudante feito no Brasil para o EF, entre 2004 e 2013, percebe-se o crescimento a cada ano, variando entre $3 \%$ e $24 \%$ nos os AI e AF, chegando a mais de R\$ 5.000,00 investidos por aluno em 2013, para ambos os níveis. No mesmo período, a nota do Indicador de Desenvolvimento da Educação Básica (IDEB) nacional também resultou positivamente, onde para os AI, subiu de 3,6 para 4,9; e de 3,2 para 4,0 nos AF. No estado de São Paulo, o investimento educacional por aluno do EF variou entre R\$ 3.572,52 e R\$ 4.426,76 (2008 a 2013). A nota do IDEB para os AI atingiu a pontuação de 5,8; e para os AF a pontuação 4,4 em 2013 (INEPa, 2016).

A educação sempre foi considerada uma variável importante na mensuração do grau de desenvolvimento de países e regiões (FRIO et al., 2018). Devido aos impactos sociais, políticos e econômicos, que podem ser causados à sociedade ou região, quando os recursos em educação são mal geridos e pela busca da qualidade do gasto público em educação, destaca-se a importância da realização de estudos sobre o tema. Uma vez que, a qualidade do gasto público interfere na qualificação do cidadão perante o mercado de trabalho, gerando aumento da produtividade do trabalho e aperfeiçoamento, ampliando sua cultura geral e análise crítica. Consequentemente, contribui para o crescimento econômico e sustentável, impactando na melhoria e distribuição de renda, saúde, emprego, participação política do cidadão e desenvolvimento social (TARDA; RODRIGUES, 2015).

Os AI do EF merecem maior atenção por parte dos gestores e governantes, devido à influência do desempenho das instituições de Educação Básica com a qualidade alcançada pelas instituições do ensino superior (GRAMANI; DUARTE, 2011). Desta forma, ao longo do presente estudo, foram listados alguns trabalhos realizados nos últimos anos sobre a eficiência do gasto público em educação no Brasil, englobando a mesma metodologia aplicada ao presente estudo, com o intuito de analisar a abrangência, os indicadores, o modelo e as técnicas estatísticas selecionadas.

Estes estudos embasaram a elaboração de um modelo para mensurar a eficiência dos 29 municípios da microrregião de Piracicaba, abrangendo oito diretorias de ensino do estado de São Paulo. Para isso, a metodologia aplicada foi a Análise Envoltória de Dados (Data Envelopment Analysis - DEA), que é uma técnica não-paramétrica, que mede a eficiência dos municípios analisados, perante seus insumos (Investimento por aluno no EF) e produtos (Nota do IDEB de AI e AF do município). Complementarmente, buscando identificar fatores influenciadores da eficiência obtida por cada município, foram utilizadas técnicas estatísticas, como regressão linear e correlação, juntamente com índices e dados sociais de instituições governamentais.

$\mathrm{O}$ estudo foi dividido em cinco seções, primeiro foram apresentadas informações sobre o cenário educacional no Brasil e o impacto da qualidade do gasto público em educação na sociedade; na segunda parte, a revisão da literatura, abordando os temas de gasto público e a importância da eficiência deste, e a explanação sobre a metodologia DEA; na terceira seção estão os procedimentos metodológicos e como estes foram aplicados aos dados coletados, juntamente com as técnicas estatísticas; na sequência estão os resultados obtidos e análises sobre as eficiências e predito; e por fim, as conclusões, sugestões e limitações do estudo realizado. 


\section{Aspectos Teóricos}

\subsection{Qualidade do Gasto Público}

Desde os primórdios da civilização, a arrecadação de impostos nunca foi bem vista pelos pagadores, e isso se deve a relação entre o custo e o benefício jamais visto por estes, onde a percepção existente era de exploração dos ganhos familiares. Com o passar do tempo, o desbravamento de novas áreas, o comércio e a industrialização, os governantes perceberam a necessidade de organizar a sociedade, aumentando o número de impostos, mas realizando dispêndios em infraestrutura e condições sociais.

Devido às origens históricas do gasto público, existe a sensação de que este nunca retorna a sociedade, na mesma proporção em que foi arrecadado. Segundo Pires (2008) a qualidade do gasto público é uma decorrência do controle exercido sobre ele. Como, de resto, acontece também no caso dos gastos privados - pessoais, familiares, empresariais. E, define o gasto público como o gasto estatal ou governamental, financiado com recursos auferidos compulsoriamente. Onde, estes possuem dimensões operacionais baseadas na economicidade (eficiência e eficácia), legitimidade (efetividade e transparência) e legalidade, e ainda estão ligados a fatores financeiros, sustentáveis e políticos (Pires 2008). Assim, quando é abordada a questão de gasto público, é necessária a definição de qualidade do gasto público, onde a palavra qualidade pode significar eficiência ou a virtude ou característica de se conseguir o melhor rendimento com o mínimo de erro e/ou dispêndios de energia, tempo, dinheiro ou meios (MAIA et al., 2007).

Abordando a eficiência do gasto público, Cândido Junior (2001) explana que o gerenciamento de recursos finitos com necessidades infinitas, é desafiador, devido à alocação destes recursos, e que a "sociedade espera do poder público a melhor utilização dos recursos, uma vez que, existem limites para a expansão das receitas que financiam o aumento dos gastos per capita".

No tocante ao gasto público em educação no Brasil, o estudo feito por Diaz (2012) examinou os gastos do país com educação, mostrando que estes gastos, em termos absolutos são baixos, mas se comparados ao Produto Interno Bruto (PIB), o Brasil aparece entre os países que mais gastam com este setor, sendo $6,6 \%$ da renda nacional. Porém, mesmo com este dado em avaliações de desempenho, identifica-se uma falha no ensino. Destaca ainda, que:

"maiores gastos não necessariamente são acompanhados por mudanças em aspectos que afetam diretamente as condições ou fatores que efetivamente são responsáveis pela melhoria da qualidade do ensino público municipal. Assim, antes de elevar gastos é preciso identificar com a devida precisão quais aspectos devem ser alterados, e somente, então, avaliar quais recursos seriam necessários para promover as transformações necessárias. Avaliações de resultados devem ser feitas constantemente para verificar se os objetivos estão sendo alcançados" (DIAZ, 2012).

Neste sentido, Silva e Almeida (2012) afirmam que os gestores municipais procuram justificar o fraco desempenho dos indicadores educacionais relacionando-os com a limitação dos recursos recebidos. Em compensação, os pesquisadores argumentam que o fraco desempenho do ensino público municipal deve-se à ineficiência na aplicação dos recursos.

Com base em diversos fatores, a Organização das Nações Unidas (UNESCO) ressalta que a qualidade e igualdade para o Brasil ainda são desafios essenciais a cumprir para que o país possa atender as necessidades da construção da sociedade do conhecimento. Em seu relatório de 2016, a UNESCO, apresentou dados de que 58 milhões de crianças estão fora da escola no mundo e cerca de 100 milhões não completarão a escola primária. Desta forma, justifica-se cada vez mais, a necessidade de estudos de eficiências em gasto público em educação e para resultar em um ensino público de qualidade.

\subsection{Análise Envoltória de Dados (DEA)}

A metodologia da Análise Envoltória de Dados (Data Envelopment Analysis - DEA) é aplicada para o cálculo da eficiência das unidades de tomadoras de decisão (Decision Making Units - DMU), foi introduzido na literatura por Charnes, Cooper e Rhodes em 1978, através do modelo CCR (Constant Returns to Scale - CRS), onde este constrói uma superfície linear por partes, não paramétrica, envolvendo os dados. Trabalha com retornos constantes de escala, isto é, qualquer variação nas entradas (inputs) produz variação proporcional nas saídas (outputs) (MELLO et al. 2005). 
Esta, também é chamada de análise de fronteiras, é considerada uma técnica relativamente nova e de ampla aplicabilidade para diversos problemas no mundo real. É uma técnica multivariável, que compara os dados de entrada e saída através da eficiência das unidades analisadas, estabelecendo indicadores e avaliando a eficiência (COLIN, 2013). O método compara as DMU's, diferenciando-as pelos recursos utilizados (inputs) e bens produzidos (outputs), resultando em pesos ou coeficientes que serão obtidos através de Programação Linear (SAVIAN; BEZERRA, 2013; COLIN, 2013).

Sendo o DEA uma técnica que mede a eficiência, há necessidade de definir os conceitos de eficácia, eficiência e efetividade os quais são fundamentais para o entendimento dessa ferramenta. A eficácia remete a condições controladas e a resultados desejados, é a capacidade que a unidade produtiva tem de atingir os objetivos estabelecidos (MELLO et al., 2005). A eficiência pode ser definida como a competência para se produzir resultados com dispêndio mínimo de recursos; define-se sendo "boa utilização" dos recursos. O DEA considera o máximo que poderia ter sido produzido das unidades produtivas. A efetividade examina em que medida são promovidos os resultados do projeto; é a capacidade atingir objetivos (MARINHO; FAÇANHA, 2001).

Em 1984, os autores Banker, Charnes, e Cooper, criaram um modelo similar, o BCC (Variable Returns of Scale - VRS), mas este possui os retornos de escala variáveis, isto é, substitui o axioma da proporcionalidade entre inputs e outputs pelo axioma da convexidade. Ao obrigar que a fronteira seja convexa, o modelo BCC permite que DMU's que operam com baixos valores de inputs tenham retornos crescentes de escala e as que operam com altos valores tenham retornos decrescentes de escala (MELLO et al., 2005).

Existem outros modelos DEA, desenvolvidos ao longo do tempo, mas estes dois são os mais utilizados nas aplicações nacionais e internacionais, e a modelagem matemática destes, é diferenciada apenas por uma restrição. As diferenças entre os modelos CRS e VRS, visualizam-se na formulação matemática, e na exemplificação gráfica na seleção das DMU's eficientes pela constância ou variação da reta, conforme representado na Figura 01.

Figura 1 - Modelos DEA-CRS e VRS.

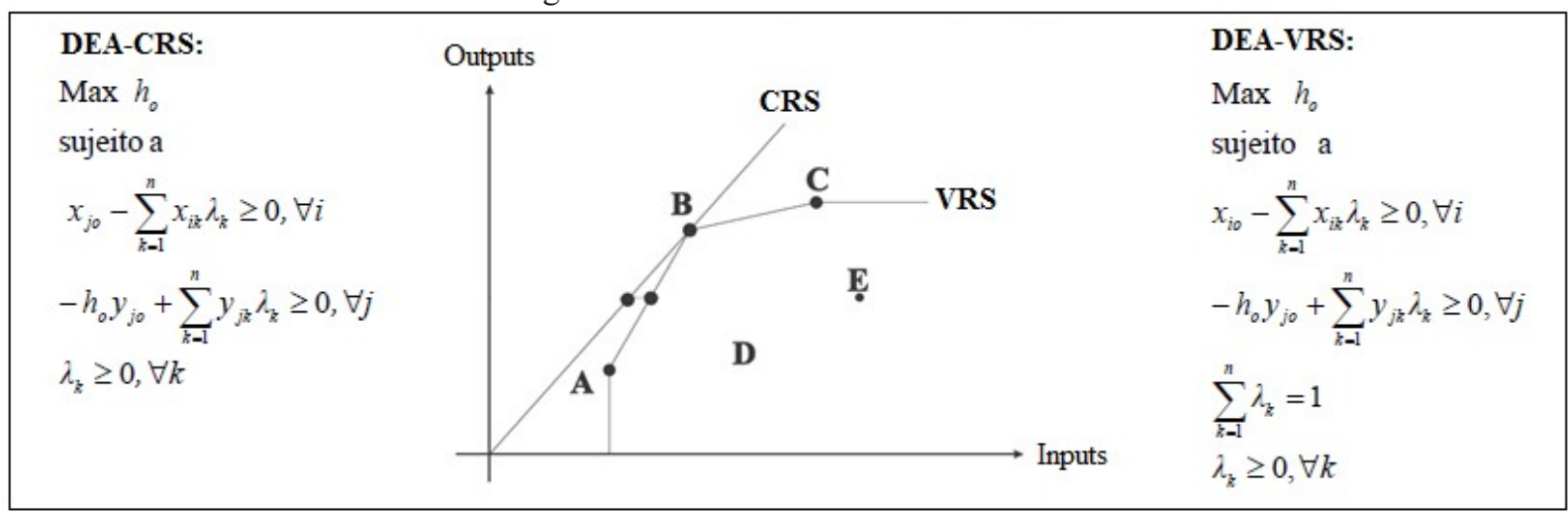

Fonte: Adaptado de Mello et al. (2005) e Gramani e Duarte (2011).

Como as DMU's representam as operações ou processos da empresa, cada uma tem uma configuração de inputs e outputs, representando a multiplicação do desempenho. Considerando n observações em cada DMU $(j=1, \ldots, n)$, usa-se $\mathrm{m}$ inputs $x_{i j}(i=1,2, \ldots, m)$ para produzir $y_{r j}(r=1,2, \ldots, s)$. Os modelos CRS e VRS diferenciam-se na formulação matemática, uma vez que no segundo é acrescido a restrição $\sum_{j=1}^{n} \lambda_{j}=1$ (Figura 1).

Para a escolha dos inputs e outputs deve-se ter atenção, respeitando-os como insumos e produtos respectivamente. A quantidade de DMU's é definida a partir do cálculo que considera apto para análise no DEA, aquele que possui um número maior ou igual à multiplicação das variáveis de inputs (I) e outputs (O), multiplicados por dois, ou seja, 2x (I x O) (DYSON et al., 2001; COOK et al., 2014). 


\subsubsection{Análise das Licitações Monitoradas e Intervenções}

Desde os anos 2000, a temática sobre educação e a eficiência do gasto público vem sendo estudada por diversos autores nacionais e internacionais com aplicação da metodologia DEA, conforme a revisão bibliográfica elaborada por Frio et al. (2018), que descreve os estudos de Mancebon e Molinero (2000), Mizala et al. (2002), Sutherland, Price e Gonand (2007), Delgado e Machado (2007), Hu et al. (2009), Silva e Almeida (2012), Scarpin et al. (2012), Savian e Bezerra (2013), Wilbert e D’Abreu (2013), Gonçalves e França (2013), Diel et al. (2014) e Macêdo et al. (2015). Com essa revisão, os autores concluíram que a grande maioria das evidências nacionais observa níveis elevados de ineficiência, demonstrando assim a dificuldade dos gestores públicos em alocar de forma eficiente os recursos disponíveis (FRIO et al., 2018).

Diversos estudos foram encontrados na pesquisa bibliográfica realizada em âmbito nacional sobre o gasto público em educação e a aplicação da metodologia DEA, onde foram selecionados apenas aqueles que utilizaram o IDEB como variável, que é o propósito do presente estudo (Tabela 01). Nota-se que, há um estudo específico para alguns estados brasileiros, e aqueles que não os tem, estão incluídos em outro estudo que abrange todos os estados. Sendo assim, pode-se afirmar que, já foram realizadas pesquisas para todos os estados brasileiros referentes ao tema proposto, mas nem todos os municípios brasileiros compõem esta estimativa, geralmente, devido à falta de dados.

Tabela 1 - Aplicações de DEA e gasto público em educação.

\begin{tabular}{|l|l|l|}
\hline \multicolumn{1}{|c|}{ Autor (es)/Modelo DEA } & \multicolumn{1}{|c|}{$\begin{array}{c}\text { Abrangência/ } \\
\text { Período }\end{array}$} & \multicolumn{1}{|c|}{ Dados } \\
\hline $\begin{array}{l}\text { Savian e Bezerra (2013)/DEA- } \\
\text { CRS }\end{array}$ & $\begin{array}{l}381 \text { municípios do } \\
\text { Paraná/2005 a 2009 }\end{array}$ & $\begin{array}{l}\text { Gasto por aluno, número de escolas municipais, } \\
\text { matrículas/professor, PIB per capita e IDEB. }\end{array}$ \\
\hline Firmino (2013)/DEA-VRS & $\begin{array}{l}149 \text { municípios da } \\
\text { Paraíba/2007 a 2009 }\end{array}$ & $\begin{array}{l}\text { Transferências do FUNDEB e outras fontes e } \\
\text { IDEB. }\end{array}$ \\
\hline $\begin{array}{l}\text { Wilbert e D'Abreu (2013)/DEA- } \\
\text { CRS e VRS }\end{array}$ & $\begin{array}{l}102 \text { municípios de } \\
\text { Alagoas/2007 a 2011 }\end{array}$ & $\begin{array}{l}\text { PIB, habitantes, estudantes, IDEB, alunos } \\
\text { matriculados e gastos com educação. }\end{array}$ \\
\hline Diniz et al. (2014)/DEA-DSBM & $\begin{array}{l}3.013 \text { municípios do } \\
\text { Brasil/2004 a 2009 }\end{array}$ & $\begin{array}{l}\text { Custo por aluno do município, Fatores } \\
\text { Socioeconômicos e Familiares, Professores e } \\
\text { Variáveis não-agrupadas, e IDEB. }\end{array}$ \\
\hline $\begin{array}{l}\text { Silva Filho } \text { et al. (2014)/DEA- } \\
\text { CRS e VRS }\end{array}$ & $\begin{array}{l}12 \text { colégios militares } \\
\text { do Brasil/2009 e } \\
2011\end{array}$ & $\begin{array}{l}\text { Despesa com educação, alunos matriculados e } \\
\text { professores, e IDEB. }\end{array}$ \\
\hline Silva et al. (2015)/DEA-CRS & $\begin{array}{l}\text { Municípios de SP, RJ } \\
\text { e BH/ 2005 a 2011 }\end{array}$ & $\begin{array}{l}\text { Despesas na função educação per capita, } \\
\text { docentes, escolas, relação docentes/alunos e } \\
\text { IDEB, outros. }\end{array}$ \\
\hline $\begin{array}{l}\text { Rodrigues } \text { et al. (2015)/DEA- } \\
\text { CRS }\end{array}$ & $\begin{array}{l}21 \text { municípios } \\
\text { mineradores de } \\
\text { Minas Gerais/2013 }\end{array}$ & $\begin{array}{l}\text { Gasto médio por aluno do EF, \% docentes com } \\
\text { curso superior, IDEB e Taxa de Distorção } \\
\text { Idade-Série. }\end{array}$ \\
\hline Sousa et al. (2016)/DEA-VRS & $\begin{array}{l}58 \text { municípios do } \\
\text { Espírito Santo/2014 }\end{array}$ & $\begin{array}{l}\text { Despesas Liquidadas, Gasto Médio por aluno e } \\
\text { IDHM (Educação e Renda) e IDEB. }\end{array}$ \\
\hline
\end{tabular}

Fonte: Elaborado pelos autores.

Nos trabalhos listados, não foi encontrada uma seleção idêntica de inputs e outputs, e também nenhum destes foram reproduzidos por outros autores integralmente. Em contrapartida, muitos inputs e outputs se repetiram, e alguns trabalhos foram baseados parcialmente em outros previamente feitos. Os modelos DEA aplicados concentraram-se no CRS e VRS. Alguns estudos aplicaram métodos estatísticos, tanto para justificar o resultando, quanto para analisar os dados coletados, onde a técnica mais aplicada foi a regressão, variando no tipo.

\section{Metodologia}

Para o presente estudo foram selecionados municípios de 08 diretorias de ensino da Secretaria Estadual de Educação do estado de São Paulo (SEE-SP), selecionando dados dos anos de 2009, 2011 e 2013. As diretorias de ensino selecionadas integram a mesorregião de Piracicaba (Figura 2): Americana, Botucatu, Capivari, Limeira, Piracicaba, Pirassununga, São Carlos e Sumaré, que juntas compreendem 65 municípios. A seleção por diretorias foi adotada devido às atribuições das diretorias de ensino que têm funções gerir o processo de ensino e aprendizagem no cumprimento das políticas públicas, diretrizes e metas de educação, atividades administrativas, financeiras e de recursos humanos, monitoramento de indicadores, dentre outras funções educacionais, portanto estabelecendo diretrizes parecidas para os municípios que constituem cada diretoria (DECENTRO, 2016). 
Figura 02 - Diretorias de ensino.

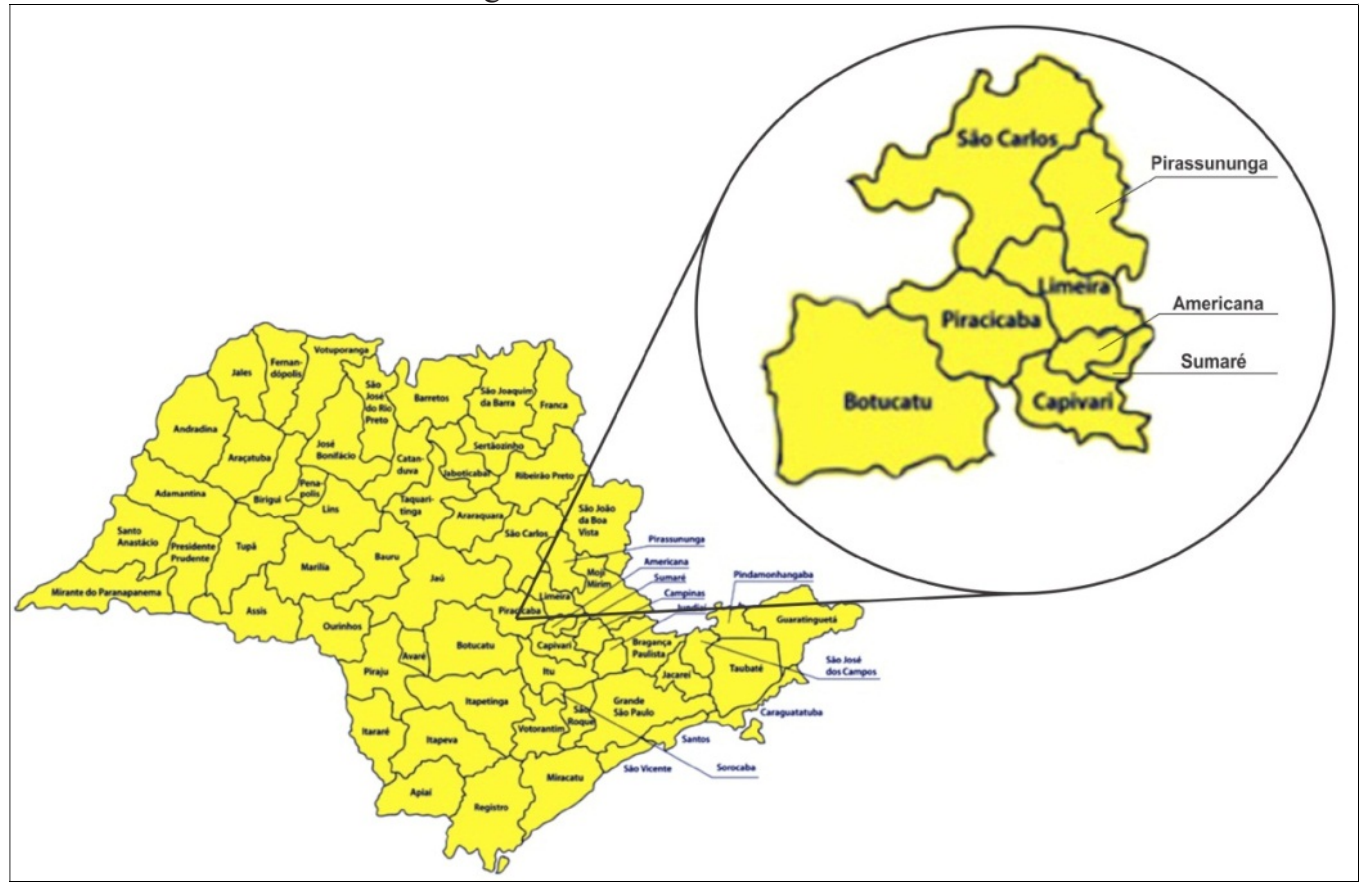

Fonte: Mapa da SEE-SP (2016) adaptado pelos autores.

Os inputs para este estudo foram coletados no item 4.2 - Investimento por aluno no EF em reais (R\$), disponíveis nos Relatórios de Indicadores do Sistema de Informação sobre Orçamentos Públicos em Educação (SIOPE), na base de dados do Fundo Nacional de Desenvolvimento da Educação (FNDE). Os anos selecionados foram entre 2008 a 2013, para cada município estudado. Com estes, foi feita a média para cada dois anos (entre: 2008 e 2009; 2010 e 2011; 2012 e 2013), para que fosse possível relacionar o investimento por aluno com as notas do IDEB de cada ano, uma vez que este é realizado a cada dois anos. Os inputs utilizados têm as siglas InvEdu09, InvEdu11 e InvEdu13.

Os outputs selecionados foram as notas do IDEB do município para os AI e os AF, disponíveis no banco de dados do Instituto Nacional de Estudos e Pesquisas Educacionais Anísio Teixeira (INEP), compreendendo os anos de 2009, 2011 e 2013 (INEPa, 2016). Destaca-se que o IDEB tem o intuito de mensurar a qualidade da educação, através do fluxo escolar e médias de desempenho nas avaliações. Este é calculado com do Censo Escolar, Sistema de Avaliação Básica (SAEB) ou Prova Brasil. Foi criado pelo INEP em 2007, onde os estudantes fazem uma avaliação ao final das etapas de ensino (AI, AF e EM). Buscando identificar e monitorar os alunos com baixo rendimento escolar, este indicador é calculado com base na fórmula: $I D E B_{j i}=N_{j i} P_{j i} ; 0 \leq N_{j}$ $\leq 10 ; 0 \leq P_{j} \leq 1$ e $0 \leq I D E B_{j} \leq 10$. Onde i é o ano do exame (SAEB e Prova Brasil) e do Censo Escolar; $N_{j i}$ é a média da proficiência em Língua Portuguesa e Matemática (entre 0 e 10), dos alunos da unidade $j$, obtida em determinada edição do exame realizado ao final da etapa de ensino; $P_{j i}$ é o indicador de rendimento baseado na taxa de aprovação da etapa de ensino dos alunos da unidade $j$ (INEPb, 2016). Os outputs utilizados terão as siglas IDEB-I09, IDEB-I11 e IDEB-I13 para os AI; e IDEB-F09, IDEB-F11 e IDEB-F13 para os AF.

As variáveis inputs e outputs foram analisadas no modelo DEA-VRS orientado aos outputs, para os anos de 2009, 2011 e 2013, com os dados respectivos. Alguns municípios foram excluídos da amostra, devido à falta de dados do IDEB municipal, inexistindo dados para AI ou AF, para todos os anos analisados, restando 29 municípios.

A escolha do modelo DEA-VRS, deu-se devido este modelo comparar as DMU's de tamanhos similares. Assim, os três períodos do modelo foram analisados separadamente. O software utilizado foi o STATA ${ }^{\circledR}$, versão 2014, através o comando DEA, criado por Ji e Lee (2010).

Para explicar a eficiência obtida pelas DMU's deste estudo, buscaram-se fatores intervenientes (indicadores de educação e sociais) que justificassem os resultados alcançados, e que pudessem auxiliar aos gestores dos gastos públicos em educação na tentativa de diminuir interveniências. A variável dependente foi a eficiência obtida no DEA, e as independentes estão na Tabela 02. Ressalta-se que, não foi possível captar todas 
as variáveis para todos os anos no presente estudo, pois algumas não são elaboradas anualmente; e outras não existiam para todos os anos. Foi aplicada a Razão de Odds para as variáveis \%DocSup, IndEsfDoc, IndRegDoc, IndCGE e AdForDoc, com intuito de utilizar somente a informação positiva da variável, onde quanto maior melhor.

Tabela 02 - Variáveis Independentes

\begin{tabular}{|c|l|}
\hline Variável Independente & \multicolumn{1}{|c|}{ Descrição (Fonte) } \\
\hline PIBMpc & Produto Interno Bruto do Município per capita: 2009,2011 e 2013 (IBGE). \\
\hline MédiaA/T & $\begin{array}{l}\text { Média de Alunos por Turma no EF do Município: 2009, 2011 e 2013 } \\
\text { (INEPa). }\end{array}$ \\
\hline TaxaDis & $\begin{array}{l}\text { Taxa de Distorção Idade-Série no EF do Munícipio: 2009, 2011 e 2013 } \\
\text { (INEPa). }\end{array}$ \\
\hline TaxaRend & Taxa de Rendimento no EF do Município: 2009, 2011 e 2013 (INEPa). \\
\hline IDHM* & Índice de Desenvolvimento Humano Municipal: 2010 (Atlas Brasil). \\
\hline \%PopRur* & Percentual de População Rural do Município: 2010 (Atlas Brasil) \\
\hline TaxaFq6_14* & $\begin{array}{l}\text { Taxa de Frequência Escolar de 06 a 14 anos do Município: } 2010 \text { (Atlas } \\
\text { Brasil). }\end{array}$ \\
\hline MédHAD** & Média de Hora-Aula Diária no EF do Município: 2011 e 2013 (INEPa). \\
\hline \%DocSup** & Percentual de Docentes com Ensino Superior: 2011 e 2013 (INEPa). \\
\hline IndEsfDoc** & Indicador de Esforço do Docente: 2011 e 2013 (INEPa). \\
\hline IndRegDoc** & Indicador de Regularidade do Docente: 2013 (INEPa). \\
\hline IndCGE** & Índice de Complexidade de Gestão da Escola: 2013 (INEPa). \\
\hline AdForDoc** & Adequação da Formação do Docente: 2013 (INEPa). \\
\hline
\end{tabular}

Fonte: Dados do IBGE (2016), INEPa (2016) e Atlas Brasil (2013) adaptado pelos autores.

* Variável não elaborada anualmente. **Variável com dados faltantes.

Nas variáveis dependentes e independentes foi aplicado teste de Variance Inflation Factor (VIF), com intuito de garantir a não colinearidade destas; e na regressão linear, para explicar as interveniências na eficiência de cada município, e ainda, para a elaboração do predito da eficiência. Ambos os procedimentos foram analisados no software STATA ${ }^{\circledR}$, onde foram utilizados os comandos: vif e regress. Na sequência, foram elaboradas para exposição dos resultados.

\section{Resultados e Discussões}

Analisando os dados coletados para o DEA, foi possível observar que, dentre as cidades selecionadas, Paulínia foi a que apresentou maior valor no investimento em Educação Fundamental em média nos três anos analisados, seguida de Americana e Santa Rita do Passa Quatro, conforme Figura 3. Em contrapartida, as DMU que menos investiram foram: Araras, Laranjal Paulista e Cosmópolis. As notas do IDEB oscilaram na média para os três anos, onde a maior nota nos AI foi a de Elias Fausto, e a menor a de Bofete; e para os AF, a maior foi de Americana e a menor de Itirapina. 
Figura 03 - Dados dos inputs e outputs.

\begin{tabular}{|c|c|c|c|c|c|c|c|c|c|}
\hline Município & IDEB-I09 & IDEB-I11 & IDEB-I13 & IDEB-F09 & IDEB-F11 & IDEB-F13 & InvEdu09 & InvEdu11 & InvEdu13 \\
\hline 1 Americana & 6,4 & 6,1 & 6,5 & 5,4 & 5,6 & 5,7 & 4744,31 & 5949,52 & 8390,345 \\
\hline 2 Analândia & 5,6 & 4,9 & 5,1 & 4,3 & 4,7 & 5,0 & 4849,22 & 5378,46 & 5604,77 \\
\hline 3 Anhembi & 5,5 & 5,1 & 5,5 & 4,6 & 4,4 & 4,5 & 4485,33 & 5292,25 & 5793,25 \\
\hline 4 Araras & 5,7 & 5,4 & 5,9 & 4,6 & 4,5 & 5,1 & 3629,11 & 4504,38 & 7031,50 \\
\hline 5 Areiópolis & 5,3 & 5,7 & 5,7 & 4,0 & 4,1 & 4,2 & 2490,65 & 3031,11 & 4125,31 \\
\hline 6 Bofete & 5,1 & 4,8 & 4,8 & 4,2 & 4,1 & 4,4 & 3657,46 & 4473,41 & 5355,05 \\
\hline 7 Botucatu & 5,9 & 5,6 & 6,0 & 4,4 & 4,4 & 4,5 & 4326,17 & 4705,08 & 6354,88 \\
\hline 8 Capivari & 5,5 & 5,7 & 5,9 & 5,1 & 5,5 & 5,2 & 3503,85 & 4253,22 & 5437,26 \\
\hline 9 Cesário Lange & 4,8 & 5,0 & 5,9 & 4,1 & 4,4 & 4,6 & 3066,59 & 3880,84 & 4674,35 \\
\hline 10 Charqueada & 5,6 & 5,8 & 6,2 & 4,6 & 4,6 & 4,5 & 3386,75 & 4067,24 & 5601,28 \\
\hline 11 Conchas & 5,4 & 5,7 & 6,0 & 4,1 & 4,5 & 4,7 & 2975,03 & 3620,52 & 4678,21 \\
\hline 12 Corumbatai & 5,7 & 6,1 & 4,8 & 4,6 & 4,3 & 4,5 & 3700,50 & 4307,33 & 6811,06 \\
\hline 13 Cosmopolis & 5,5 & 5,3 & 5,5 & 4,9 & 5,7 & 5,0 & 2733,87 & 3554,38 & 4647,32 \\
\hline 14 Descalvado & 5,4 & 5,2 & 5,6 & 4,6 & 4,8 & 4,1 & 3207,59 & 3803,22 & 5554,73 \\
\hline 15 Dourado & 5,6 & 5,8 & 5,1 & 4,7 & 5,0 & 4,9 & 2940,33 & 3925,87 & 4684,10 \\
\hline 16 Elias Fausto & 6,5 & 5,9 & 6,8 & 6,0 & 5,0 & 5,0 & 3551,07 & 4166,84 & 4587,41 \\
\hline 17 Itatinga & 4,6 & 4,9 & 5,4 & 4,3 & 3,8 & 3,8 & 3760,64 & 4921,61 & 5434,63 \\
\hline 18 Itirapina & 5,6 & 5,5 & 5,6 & 3,9 & 3,5 & 4,0 & 3562,77 & 5903,93 & 7276,09 \\
\hline 19 Laranjal Paulista & 5,5 & 5,4 & 5,3 & 4,7 & 4,8 & 4,8 & 2876,58 & 3325,20 & 3944,47 \\
\hline 20 Mombuca & 5,4 & 5,2 & 5,2 & 4,7 & 4,9 & 4,3 & 3723,55 & 3792,91 & 4644,77 \\
\hline 21 Monte Mor & 5,4 & 5,4 & 5,6 & 4,5 & 4,6 & 5,0 & 3617,52 & 3463,72 & 3921,14 \\
\hline 22 Pardinho & 4,9 & 5,0 & 5,0 & 3,7 & 4,3 & 4,2 & 3916,11 & 4785,06 & 5838,1 \\
\hline 23 Paulínia & 5,8 & 5,6 & 5,9 & 4,8 & 4,4 & 4,1 & 7611,79 & 9861,69 & 11488,53 \\
\hline 24 Porto Ferreira & 5,3 & 5,3 & 6,1 & 5,1 & 5,1 & 4,5 & 3358,96 & 4062,22 & 4880,10 \\
\hline 25 Quadra & 5,3 & 5,1 & 5,4 & 4,6 & 4,8 & 5,2 & 3307,56 & 4976,58 & 6267,0 \\
\hline 26 Rio das Pedras & 5,5 & 5,6 & 5,6 & 5,1 & 4,7 & 4,9 & 3258,73 & 3831,91 & 4958, \\
\hline 27 Santa Gertrudes & 5,6 & 5,5 & 5,8 & 5,1 & 5,1 & 5,4 & 2955,31 & 3638,45 & 4475 \\
\hline 28 Santa Rita do Passa Quatro & 6,0 & 5,7 & 6,0 & 5,0 & 4,9 & 4,5 & 4304,23 & 6323,38 & 6799 \\
\hline 29 Sumaré & 5,3 & 5,5 & 5,7 & 5,2 & 5,2 & 4,9 & 3865,46 & 4517,57 & 5639,9 \\
\hline
\end{tabular}

Fonte: Elaborado pelos autores.

O modelo DEA-VRS orientado aos outputs teve como objetivo a maximização das notas do IDEB, assim, conforme os resultados apresentados na Figura 4 (Eficiência), no ano de 2009, três DMU's foram eficientes: Areiópolis, Elias Fausto e Cosmópolis. Para o ano de 2011, a quantidade de municípios eficientes foi superior, uma vez que, sete DMU's foram eficientes: Americana, Areiópolis, Capivari, Elias Fausto, Dourado, Cosmópolis e Corumbataí. Já para 2013, quatro atingiram a eficiência: Americana, Monte Mor, Elias Fausto e Santa Gertrudes. As demais DMU's tiveram uma variação entre $72 \%$ e $99 \%$, identificando que a métrica de resposta tem uma escala reduzida (baixa dispersão), indicando que qualquer resultado abaixo de $100 \%$, representa uma distância expressiva para alcance da eficiência para as determinadas DMU's, sendo este um resultado insatisfatório para os municípios.

A regressão resultou em um R-Quadrado Ajustado (Adj R²) para cada ano, onde em 2009 resultou em 7\%; 2011 em 31\%; e $2013 \mathrm{em} \mathrm{40 \% ,} \mathrm{significando} \mathrm{o} \mathrm{quanto} \mathrm{as} \mathrm{variáveis} \mathrm{intervenientes} \mathrm{explicam} \mathrm{as} \mathrm{eficiências} \mathrm{resultadas.} \mathrm{O}$ $\mathrm{P}$-valor considerado para as variáveis intervenientes no presente estudo foi abaixo de $10 \%$, com confiabilidade de $95 \%$. Resultando em população rural (10\%) em 2009; média de aluno por turma $(0,2 \%)$ e população rural (7\%) em 2011; e média de alunos por turma (1\%), população rural (4,1\%), docentes com nível superior $(2,2 \%)$ e índice de regularidade de docentes (7,5\%). O VIF manteve-se abaixo de 2 , indicando que não há multicolinearidade entre as variáveis utilizadas na regressão.

Com a regressão foi gerado o Predito (eficiências esperadas), disposto na Figura 4, para os 29 municípios. Comparando com as eficiências observadas, as cidades que obtiveram resultados superiores ao Predito em 2009 foram: Americana, Analândia, Areiópolis, Conchas, Corumbataí, Cosmópolis, Dourado, Elias Fausto, Laranjal Paulista, Quadra, Rio das Pedras, Santa Gertrudes e Santa Rita do Passa Quatro. No ano de 2011: Areiópolis, Capivari, Charqueada, Conchas, Corumbataí, Cosmópolis, Dourado, Elias Fausto, Laranjal Paulista, Mombuca, Santa Gertrudes e Santa Rita do Passa Quatro. E, para 2013 foram: Americana, Araras, Areiópolis, Charqueada, Corumbataí, Dourado, Elias Fausto, Laranjal Paulista, Monte Mor, Rio das Pedras e Santa Rita do Passa Quatro. Mesmo considerando os fatores estruturais, gestão educacional e gestão pública que determinariam uma eficiência menor estas cidades conseguiram proporcionar melhores resultados. 
Figura 4 - Resultados DEA e Preditos

\begin{tabular}{|l|c|c|c|c|c|c|}
\hline \multirow{2}{*}{ Município } & \multicolumn{2}{|c|}{2009} & \multicolumn{2}{c|}{2011} & \multicolumn{2}{c|}{2013} \\
\cline { 2 - 7 } & Eficiência & Predito & Eficiência & Predito & Eficiência & Predito \\
\hline Americana & $98 \%$ & $93 \%$ & $100 \%$ & $99 \%$ & $100 \%$ & $98 \%$ \\
\hline Analândia & $86 \%$ & $92 \%$ & $83 \%$ & $87 \%$ & $91 \%$ & $91 \%$ \\
\hline Anhembi & $85 \%$ & $93 \%$ & $84 \%$ & $91 \%$ & $85 \%$ & $86 \%$ \\
\hline Araras & $88 \%$ & $91 \%$ & $90 \%$ & $94 \%$ & $92 \%$ & $90 \%$ \\
\hline Areiópolis & $100 \%$ & $91 \%$ & $100 \%$ & $96 \%$ & $96 \%$ & $93 \%$ \\
\hline Bofete & $78 \%$ & $77 \%$ & $81 \%$ & $84 \%$ & $81 \%$ & $81 \%$ \\
\hline Botucatu & $91 \%$ & $91 \%$ & $92 \%$ & $93 \%$ & $89 \%$ & $91 \%$ \\
\hline Capivari & $86 \%$ & $92 \%$ & $100 \%$ & $100 \%$ & $96 \%$ & $98 \%$ \\
\hline Cesário Lange & $81 \%$ & $81 \%$ & $87 \%$ & $89 \%$ & $90 \%$ & $92 \%$ \\
\hline Charqueada & $89 \%$ & $92 \%$ & $98 \%$ & $95 \%$ & $91 \%$ & $89 \%$ \\
\hline Conchas & $92 \%$ & $85 \%$ & $98 \%$ & $90 \%$ & $92 \%$ & $92 \%$ \\
\hline Corumbataí & $88 \%$ & $89 \%$ & $100 \%$ & $89 \%$ & $81 \%$ & $77 \%$ \\
\hline Cosmópolis & $100 \%$ & $91 \%$ & $100 \%$ & $97 \%$ & $93 \%$ & $91 \%$ \\
\hline Descalvado & $88 \%$ & $89 \%$ & $92 \%$ & $94 \%$ & $82 \%$ & $85 \%$ \\
\hline Dourado & $96 \%$ & $92 \%$ & $100 \%$ & $93 \%$ & $90 \%$ & $92 \%$ \\
\hline Elias Fausto & $100 \%$ & $91 \%$ & $100 \%$ & $99 \%$ & $100 \%$ & $95 \%$ \\
\hline Itatinga & $72 \%$ & $84 \%$ & $80 \%$ & $84 \%$ & $79 \%$ & $84 \%$ \\
\hline Itirapina & $86 \%$ & $89 \%$ & $90 \%$ & $98 \%$ & $82 \%$ & $89 \%$ \\
\hline Laranjal Paulista & $96 \%$ & $85 \%$ & $98 \%$ & $96 \%$ & $96 \%$ & $93 \%$ \\
\hline Mombuca & $83 \%$ & $88 \%$ & $93 \%$ & $91 \%$ & $82 \%$ & $84 \%$ \\
\hline Monte Mor & $83 \%$ & $87 \%$ & $96 \%$ & $96 \%$ & $100 \%$ & $97 \%$ \\
\hline Pardinho & $75 \%$ & $82 \%$ & $84 \%$ & $87 \%$ & $78 \%$ & $83 \%$ \\
\hline Paulínia & $89 \%$ & $89 \%$ & $92 \%$ & $92 \%$ & $87 \%$ & $91 \%$ \\
\hline Porto Ferreira & $89 \%$ & $90 \%$ & $94 \%$ & $93 \%$ & $90 \%$ & $89 \%$ \\
\hline Quadra & $85 \%$ & $83 \%$ & $87 \%$ & $91 \%$ & $94 \%$ & $94 \%$ \\
\hline Rio das Pedras & $91 \%$ & $90 \%$ & $97 \%$ & $95 \%$ & $92 \%$ & $89 \%$ \\
\hline Santa Gertrudes & $98 \%$ & $93 \%$ & $99 \%$ & $98 \%$ & $100 \%$ & $88 \%$ \\
\hline Santa Rita do Passa Quatro & $92 \%$ & $88 \%$ & $93 \%$ & $91 \%$ & $89 \%$ & $88 \%$ \\
\hline Sumaré & $87 \%$ & $94 \%$ & $95 \%$ & $101 \%$ & $91 \%$ & $99 \%$ \\
\hline
\end{tabular}

Fonte: Elaborado pelos autores.

As cidades em que o Predito foi superior para o ano de 2009 foram: Anhembi, Araras, Bofete, Capivari, Charqueada, Corumbataí, Descalvado, Itatinga, Itirapina, Mombuca, Monte Mor, Pardinho, Porto Ferreira e Sumaré. No ano de 2011 foram: Analândia, Anhembi, Araras, Bofete, Botucatu, Cesário Lange, Descalvado, Itatinga, Itirapina, Monte Mor, Pardinho, Paulínia, Porto Ferreira, Quadra e Sumaré. E, para 2013 foram: Analândia, Bofete, Botucatu, Capivari, Cesário Lange, Cosmópolis, Descalvado, Itatinga, Itirapina, Mombuca, Pardinho, Paulínia e Sumaré. Esses municípios mesmo apresentando condições mais favoráveis os resultados foram inferiores, pode-se observar que há possibilidade de ingerência dos recursos ou descontrole dos fatores intervenientes, uma vez que, o Predito indica a porcentagem que se espera dos municípios, e estes não conseguiram atingir o esperado mesmo tendo recursos suficientes.

Nota-se que municípios como Sumaré, Bofete e Descalvado, mesmo com o passar dos anos, permanecem no mesmo grupo de DMU's que possuem um Predito superior, assim, demonstram a necessidade de mudanças na gestão educacional.

Os indicadores usados podem explicar a limitação de cada município estudado, como por exemplo, a média de alunos por turma, que indica que quanto maior a quantidade de alunos por turma, mais difícil será o ensino e o aprendizado. Outros fatores que podem justificar a ineficiência dos municípios são: localização das escolas, material disponível para o uso dos alunos (como bibliotecas, salas de informáticas, etc.), e fatores relacionados à competência e ética no processo de alocação de recursos públicos, porém o estudo se limitou a selecionar indicadores interferissem diretamente com a gestão dos recursos educacionais. 


\section{Conclusões}

O presente estudo identificou os municípios eficientes e ineficientes na aplicação dos recursos em educação fundamental, a partir dos dados selecionados de gastos por aluno e notas do IDEB para as oito diretorias de ensino do estado de São Paulo.

Com a finalidade de maximizar as notas do IDEB, destaca-se que somente o município de Elias Fausto foi eficiente nos três anos estudados, considerando os resultados obtidos e os esperados quando realizado um comparativo com ambos. Identificou-se que metade dos municípios estudados teve um predito superior ao observado. E com a regressão, os fatores intervenientes (indicadores sociais e educacionais) que apresentaram significância estatística revelaram a existência de problemas estruturais em todos os anos.

Estes fatores intervenientes influenciaram a eficiência dos municípios analisados, porque não estão no controle do gestor público que gerencia os recursos educacionais. Como exemplo, a quantidade de população rural, que necessita de transporte para ter acesso à educação, incentivo à educação devido ao abando e faltas escolares para o trabalho no campo, qualidade da educação e dos docentes e outros fatores. Assim, quanto maior for o número de residentes rurais, menor será a eficiência do município, ou seja, menor será a nota no IDEB, caso a gestão pública não busque dirimir os fatores interventores ao gasto público em educação.

Uma vez que, as variáveis intervenientes (quantidade de população rural, quantidade de alunos por turma, docentes com nível superior e a regularidade destes no processo educacional) forem identificadas e tratadas nos municípios estudados, pode-se obter melhoria na aplicação dos recursos em educação e, consequentemente, na melhoria na qualidade do ensino.

Este estudo diferencia-se dos demais que aplicaram a ferramenta DEA, uma vez que, através dos indicadores sociais e educacionais, conseguiu-se justificar até 40\% da eficiência alcançada em 2013, por estes. Contribuindo para uma gestão pública mais eficiente, o estudo destacou a importância da utilização de fatores intervenientes em pesquisas, para que o processo de gasto público em educação nos municípios seja mais efetivo.

Como limitação, identificaram-se alguns dados faltantes no período estudado, sendo necessária a exclusão de 36 municípios, impossibilitando a avaliação completa das oito diretorias selecionadas. E, para trabalhos futuros, recomenda-se a elaboração de um estudo similar por escolas, buscando a identificação de fatores estruturais dentro de cada município.

\section{REFERÊNCIAS}

ATLAS BRASIL. Atlas do Desenvolvimento Humano no Brasil: IDHM 2010. Disponível em: $<$ http://www.atlasbrasil.org.br/2013/pt/consulta/>. Acesso em: 15 jan. 2016.

BANKER, R. D.; CHARNES, A.; COOPER, W. W. Some models for estimating technical and scale inefficiencies in data envelopment analysis. Management Science, v. 30, n. 9, p: 1078-1092, 1984.

CÂNDIDO Jr., J. O. (2001). Os gatos públicos no Brasil são produtivos? Texto para discussão n. 977. Brasília: IPEA. Disponível em: < http://www.ipea.gov.br/ppp/index.php/PPP/article/view\%20File/77/88>. Acesso em 05 fev. 2016.

CHARNES, A.; COOPER, W. W.; RHODES, E. Measuring the efficiency of decision making units. European Journal of Operational Research, v. 2, n.6, pp. 429-444, 1978.

COLIN, E. C. Pesquisa Operacional: 170 Aplicações em Estratégia, Finanças, Logística, Produção, Marketing e Vendas. Ed. LTC: Rio de Janeiro, 2013.

COOPER, W. W; SEIFORD, L. M.; ZHU, J. Handbook on Data Envelopment Analysis. Second Edition. Springer, New York, 2011. 
COOK,W. D.; TONE, K.; ZHU, J. Data envelopment analysis: Prior to choosing a model. Omega, v. 44, pp. 1$4,2014$.

DIAZ, M. D. M. Qualidade do gasto público municipal em ensino fundamental no Brasil. Revista de Economia Política, v. 32, n. 1 (126), pp. 128-141, 2012.

DINIZ, J. A.; CORRAR, L. J.; LIMA, S. C. A influência das transferências condicionais na eficiência da educação fundamental brasileira. VIII Congresso Anpcont, Rio de Janeiro, 2014.

DECENTRO. Diretoria de Ensino - Região Centro: Quem somos. Disponível em: $<$ http://decentro.educacao.sp.gov.br/quem-somos>. Acesso em: 06 dez. 2016.

DYSON, R.G.; ALLEN, R.; CAMANHO, A. S.; PODINOVSKI, V. V.; SARRICO, C. S.; SHALE, E. A. Pitfalls and protocols in DEA. European Journal of Operational Research, v. 132, pp. 245-259, 2011.

FIRMINO, R. G. Avaliação da eficiência na aplicação dos recursos públicos da educação básica: um estudo nos municípios paraibanos. Dissertação (Mestrado). Universidade Federal do Rio Grande do Norte (UFRN). João Pessoa, PB, 2013.

FRIO, G. S.; FOCHEZATTO, A.; TRIACA, L. M.; FINN, E. S.; BRAATZ, J. Eficiência na educação: uma análise por escola no Rio Grande do Sul utilizando o método DEA em dois estágios. Revista Brasileira de Estudos Regionais e Urbanos (RBERU), v. 12, n. 1, pp. 74-89, 2018.

GRAMANI, M. C. N.; DUARTE, A. L.C. M. O impacto do desempenho das instituições de educação básica na qualidade do ensino superior. Ensaio: aval. pol. públ. Educ., Rio de Janeiro, v. 19, n. 72, pp. 679-702, 2011.

IBGE. Instituto Brasileiro de Geografia e Estatística. Produto Interno Bruto dos Municípios (PIB). Disponível em: $<$ http://www.ibge.gov.br/home/estatistica/pesquisas/pesquisa_resultados.php?id_pesquisa=46>. Acesso: 15 jan. 2016.

INEPa. Instituto Nacional de Estudos e Pesquisas Educacionais Anísio Teixeira. Indicadores Educacionais. Disponível em: <http://portal.inep.gov.br/indicadores-educacionais $>$. Acesso: 15 jan. 2016.

INEPb. Instituto Nacional de Estudos e Pesquisas Educacionais Anísio Teixeira. O que é o IDEB? Disponível em: $\quad<\underline{h t t p}$ ://download.inep.gov.br/educacao basica/portal_ideb/o_que_e_o_ideb/ Nota_Tecnica_n1_concepcaoIDEB.pdf>Acesso em: Acesso: 15 jan. 2016.

JI, Y.; LEE, C. Data envelopment analysis. The Stata Journal, v.10, n. 2, pp. 267-80, 2010.

MAIA, A.; VAlle, A.; Frossard, L. B.; CAMPOS, L. K.; MElO, L.; CARVAlHO, M. A. B. A importância da melhoria da qualidade do gasto público no Brasil: Propostas práticas para alcançar este objetivo. II Congresso CONSAD de Gestão Pública, Painel 32, 2007.

MARINHO, A.; FAÇANHA, L. O. Programas sociais: efetividade, eficiência e eficácia como dimensões operacionais da avaliação. Rio de Janeiro: IPEA, 2001. (Texto para discussão, n. 787). Disponível em: $<$ http://www.ipea.gov.br/pub/td/td_2001/td0787.pdf> . Acesso em: 20 jan. 2016.

MEllo J. C. C. B. S.; MEZA. L. A.; GOMES E. G.; NETO L. B. Curso de Análise de Envoltória de Dados. XXXVII Simpósio Brasileiro de Pesquisa Operacional, Gramado, RS, 2005.

PIRES, V. A. Gestão orçamentária e qualidade do gasto público. Disponível em: $<$ https://professorpires.files.wordpress.com/2012/09/gestorcgastopublbiz.pdf $>$. Acesso: 05 fev. 2016.

PORTAL BRASIL. Saiba como é a divisão do sistema de educação brasileiro. Disponível em: $<$ http://www.brasil.gov.br/educacao/2014/05/saiba-como-e-a-divisao-do-sistema-deeducacaobrasi leiro/view>. Acessado: 20 abr. 2016.

POKER JR., J. H.; NUNES, R. C.; NUNES, S. P. P. Uma avaliação de efetividade e eficiência do gasto em educação em municípios brasileiros. Cad. Fin. Públ., Brasília, n. 13, p. 263-287. 2013. 
RODRIGUES, A. C.; SOUZA, C. R.; TEIXEIRA, F. A.; CAMPOS, M. S.; BORGES, R. E. Avaliação da eficiência da aplicação dos recursos em educação dos municípios mineradores de Minas Gerais. Congresso Internacional de Administração. Ponta Grossa, PR, 2015.

SALGADO JUNIOR, A. P.; NOVI, J. C.; FERREIRA, J. Práticas escolares e desempenho dos alunos: uso das abordagens quantitativa e qualitativa. Educação \& Sociedade, v. 37, n 134, pp. 217-243, 2016.

SAVIAN, M. P.; BEZERRA, F. M. Análise de eficiência dos gastos públicos com educação no ensino fundamental no estado do Paraná. Economia \& Região, Londrina (PR), v.1, n.1, pp. 26-47, jan./jul, 2013.

SEE-SP. Secretaria Estadual de Educação do estado de São Paulo. Diretorias de Ensino. Disponível em: $<$ http://www.educacao.sp.gov.br/central-de-atendimento/Index Mapas Dir.asp >. Acesso: 05 fev. 2016.

SILVA FILHO, G. M.; PERREIRA, T. R. L.; DANTAS, M. G. S.; ARAÚJO, A. O. Análise da eficiência nos gastos públicos com educação fundamental nos colégios militares do exército: evidência para os anos de 2009 e 2011. XIV Congresso USP de Contabilidade e Controladoria. São Paulo, SP, 2014.

SILVA, J. L. M.; ALMEIDA, J. C. L. Eficiência no gasto público com educação: uma análise dos municípios do Rio Grande do Norte. Planejamento e Políticas Públicas, n. 39. 2012.

SILVA, M. C.; SOUZA, F. J.V.; BORGES, E. F.; ARAÚJO, A. O.; SILVA, J. D. G. Avaliação da função educação nos municípios de São Paulo, Rio de Janeiro e Belo Horizonte: mudança ou inércia social? CONTEXTO, Porto Alegre, v. 15, n. 29, pp. 17-29, 2015.

SIOPE. Sistema de Informação sobre Orçamentos Públicos em Educação. Indicadores Financeiros e Educacionais. Disponível em: $<$ https://www.fnde.gov.br/siope/indicadoresFinanceiros/ EEducacionais $>$. Acesso em: 05 jan. 2016.

SOUSA, W. D.; MAGALHÃES, M. A.; NASCIMENTO, J. C. H. B.; BERNARDES, J. R. Análise dos gastos na alocação dos recursos públicos destinados ao ensino fundamental dos municípios do Espirito Santo. Revista Gestão.Org v.14, n2, 2016.p.381-392.

TARDA, K. B.; RODRIGUES, R. V. A influência dos gastos em educação no crescimento econômico da região administrativa de Campinas. Estudo\& Debate, Lajeado, v. 22, n. 2, pp. 07-31, 2015.

UNESCO. Organização das Nações Unidas. Qualidade educacional no Brasil. Disponível em: $<$ http://www.unesco.org/new/pt/brasilia/education/educational-quality/>. Acesso em: 28 mar. 2016.

WILBERT, M. D.; D’ABREU, E. C. C. F. Eficiência dos gastos públicos na educação: análise dos municípios do estado de alagoas. Advances in Scientificand Applied Accounting, v. 6, n. 3, p. 348-372, 2013.

ZOGHBI, A. C.; MATTOS, E.; ROCHA, F. R.; ARVETE, P. Uma análise da eficiência nos gastos em educação fundamental para os municípios paulistas. Planejamento e políticas públicas, ppp, n. 36, jan/jun. 2011.

ZHU, J. Quantitative Models for Performance Evaluation and Benchmarking: Data Envelopment Analysis with Spreadsheets. Springer, Second Edition, New York, 2009. 